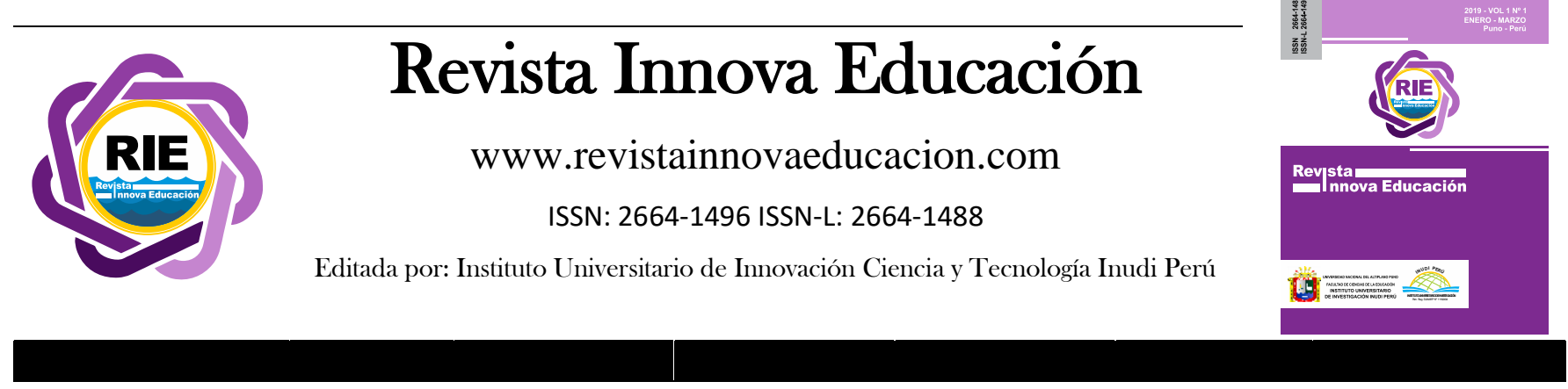

\title{
Contribución del aprendizaje invertido en la enseñanza del idioma inglés en un bachillerato mixto
}

\section{Contribution of inverted learning in English language teaching in a mixed higher education}

\author{
Juan Zamudio-Hermida ${ }^{1}$; ; José Noriega-Guerrero $^{2}$ iD; José Soto-Ortiz $^{3}$ iD \\ DOI: https://doi.org/10.35622/j.rie.2019.04.004
}

\footnotetext{
${ }^{1}$ Instituto Universitario Veracruzano, México.

${ }^{2}$ Escuela de Bachilleres "Constitución de 1917”, México.

${ }^{3}$ Universidad Pedagógica Veracruzana, México.
}

Recibido el 06/10/2019/ Aceptado el 19/10/2019/

ARTÍCULO ORIGINAL
PALABRAS CLAVE
Aprendizaje
invertido,
aprendizaje,
enseñanza,
bachillerato.

\section{KEYWORDS}

Inverted learning, learning, teaching, high school.

\begin{abstract}
En este artículo se presenta una experiencia pedagógica para la enseñanza de un segundo idioma en un bachillerato público estatal de escolaridad mixta, modalidad en la que los estudiantes acuden un día a la semana para atender clases presenciales complementando el aprendizaje de manera autónoma durante los días restantes. El objetivo fue analizar la contribución del aula invertida como estrategia de enseñanza del idioma inglés. El estudio fue de corte cuantitativo descriptivo con diseño cuasiexperimental, cuya variable se basa en el aprovechamiento académico en la asignatura de lengua adicional al español (inglés I) de un grupo de estudiantes que cursaron el primer semestre de un bachillerato público en la modalidad mixta. De manera complementaria, se realizó una entrevista al docente titular de la materia. Los resultados obtenidos señalan un $71 \%$ en las actitudes positivas acerca de la adopción de esta didáctica, lo que permitió el reforzamiento de las temáticas del curso, así como la aceptación favorable por parte del docente en el uso de esta metodología de enseñanza.
\end{abstract}

This article presents a pedagogical experience for teaching a second language in a state public high school of mixed schooling, in this modality the students attend one day a week to attend face-to-face classes complementing the learning autonomously during the remaining days. The objective was to analyze the contribution of the inverted classroom as an English language teaching strategy. The study was descriptive quantitative with a quasi-experimental design, whose study variable is based on academic achievement in the subject of additional language to Spanish (English I) of a group of students who completed the first semester of a public baccalaureate in the mixed modality. In addition, an interview was held with the teacher in charge of the subject. The results obtained indicate $71 \%$ in the positive attitudes about the adoption of this didactic, which allowed the reinforcement of the topics of the course, as well as the favorable acceptance by the teacher in the use of this teaching methodology. 


\section{INTRODUCCIÓN}

Es innegable que las tecnologías de la comunicación e información han permeado en el ámbito educativo, ya que ofrecen un abanico de aplicaciones digitales para su aprovechamiento en el aula. No obstante, este aprovechamiento depende en gran medida de las destrategias aplicadas en el aula, de los insumos disponibles y del nivel educativo de enseñanza. Innovar con recursos tecnológicos resulta un reto, por lo cual se deben utilizar los métodos de enseñanza acordes a los objetivos de cada temática.

De este modo, los nuevos cambios educativos apuntan a una enseñanza centrada en el estudiante, orientados a la participación activa, la colaboración y el desarrollo de habilidades cognitivas de acuerdo con la taxonomía de Bloom (1973) tales como: conocer, comprender, aplicar, analizar, sintetizar, evaluar, crear, etc. Lo anterior propicia que el docente se adapte a los nuevos requerimientos como es el uso de la tecnología para el desarrollo y reforzamiento del aprendizaje en las clases. Por ello, se requiere de un cambio en las estrategias de enseñanza como el aprovechamiento de las TIC para el soporte del aprendizaje.

La migración desde un modelo educativo tradicional a uno soportado por TIC, implica el rediseño del ambiente de aprendizaje que permita la transición del contexto educativo hacia la adaptación de la nueva didáctica, pero sobre todo donde el estudiante sea quien asuma el rol protagónico y se auto organice para el desarrollo de su desempeño académico, sin dejar atrás aquellas competencias necesarias para dicho auto aprendizaje (González et al., 2017). Por su parte, el docente debe considerar cuál es la mejor condición para transmitir los conocimientos, y así conseguir que el discente asimile debidamente los contenidos temáticos, aprovechando el tiempo en la construcción de su aprendizaje (González y Huerta, 2019).

Ante estos preceptos, la Dirección de la Escuela de Bachilleres Constitución de 1917 modalidad mixta, ubicada en la zona centro de la ciudad de Xalapa, Veracruz, en conjunto con un grupo de docentes, se dieron a la tarea de investigar cómo reforzar el aprendizaje del idioma inglés como segunda lengua en los estudiantes de bachillerato, ante la problemática del índice de reprobación en dicha asignatura debido a las condiciones propias del sistema mixto.

La característica principal de esta modalidad, consiste en sesionar las clases solamente el día sábado, siendo el único espacio en donde los educandos asisten a clases; asimismo, el proceso de enseñanza-aprendizaje está enfocado a los estudiantes que por sus condiciones personales no pueden asistir a clases durante la semana, tal como sucede con los bachilleratos en modalidad escolarizada tradicional. Dentro los modelos revisados para la incorporación de las TIC, el que más se adaptó a las características propias del bachillerato de tipo mixto en mención fue el denominado aula invertida, debido a las oportunidades que presenta y que son aplicables.

En este sentido, el aula invertida es un modelo pedagógico que consiste en girar los roles respecto al modelo tradicional; en el caso del estudiante debe de asumir un rol más activo en su aprendizaje, lo que implica consultar los materiales educativos el tiempo que no asiste a clases desde cualquier lugar, para que el tiempo de clase presencial sea aprovechado al máximo como por ejemplo resolver dudas de los materiales consultados, optimizando el tiempo en su aprendizaje.

Por su parte, el docente, actúa de tutor, diseñando los contenidos de aprendizaje poniéndolos a la disposición del estudiante a través de la plataforma destinada para ello, asimismo, 
debe de estar pendiente en la resolución de dudas, así como del seguimiento permanente de los foros realizados y actividades realizadas para tal fin. De acuerdo con lo anterior, el objetivo de este documento es analizar la contribución del aula invertida en la didáctica para la enseñanza del idioma inglés en un bachillerato público del estado de Veracruz.

\section{MARCO REFERENCIAL}

Los orígenes del aula invertida (flipped classroom, en inglés) provienen de cuando Bergmann y Sams (2014) videograbaron sus clases de química y los subieron a Internet mediante la herramienta vodcast para compartirlos con aquellos estudiantes que no estuvieron en clase y por ende fueran capaces de ir al día con las clases y no se vieran perjudicados por las inasistencias. De este modo, los estudiantes veían los contenidos temáticos en los vídeos y posteriormente en las sesiones presenciales se destinaron para hacer las prácticas de laboratorio, en tanto que los profesores los asistían con las dudas que fueron surgiendo. Sin embargo, Bergmann y Sams (2014) se dieron cuenta que no solamente utilizaban los vídeos sus educandos, sino que ya los comenzaban a ver otros estudiantes.

En este sentido, Bergmann y Sams (2014) argumentan que el aula invertida o el aprendizaje invertido es un enfoque pedagógico en el que la instrucción directa parte del análisis del material previamente estudiado por el discente de forma colectiva sea aplicada de manera individual y el espacio resultante se transforme en un ambiente de aprendizaje dinámico en el que el docente sea el guía en la aplicación de los conceptos de manera creativa. En un estudio realizado por Martínez y Esquivel (2018) ubican al aprendizaje invertido tomando en cuenta los elementos de los entornos mixtos de aprendizaje (blenden learning, en inglés) como un sub modelo de éste, por lo que las características y filosofía que tiene el modelo de aprendizaje mixto, son retomadas para el aprendizaje invertido aplicando una didáctica diferente, Madrid (2017).

Uno de los principales atributos del aprendizaje invertido es resultado de adoptar los conceptos que tiene la modalidad b-learning, es por ello que su diseño didáctico está basado en las diferentes teorías del aprendizaje, entre los que destaca el constructivismo en donde los roles del profesor y el estudiante están perfectamente definidos, siendo este último el responsable directo de su aprendizaje (Aguirre, 2017). Esto no implica que el catedrático no se involucre, por el contrario, el rol del docente en planteado desde el punto de vista del constructivismo, debe incidir en una interacción dialéctica entre los saberes del profesor y los del alumno, de tal manera que se llegue a la comprensión de los contenidos establecidos, así como las didácticas que propicien el aprendizaje significativo (Asens, 2015; Caraguay, 2017).

Lo anterior se confirma, en el estudio realizado por Martínez y Esquivel (2018) señalando que el eje principal del aprendizaje invertido, se sitúa bajo los postulados teóricos del aprendizaje visto desde las posturas de Vigotsky y Kolb. Visto desde la perspectiva psicológica, el constructivismo hace énfasis en que el desarrollo de las funciones mentales superiores se origina desde y en el entorno social, esto es en contacto con otros individuos u objetos, principalmente mediados por el lenguaje. De modo que el aprendizaje, se basa en la interacción, esto sin dejar inadvertido que, en vías de la construcción del conocimiento, es importante considerar las experiencias previas del estudiante, así como del contexto especifico de ambos (docente y 
discente) dada la influencia que tienen las diferentes situaciones físicas, biológicas, psicológicas, sociales y culturales (Cerda, 2014).

De acuerdo con Capacho (2011) el estudiante que realiza su formación en los ambientes virtuales, al explorar el aula virtual, es común que encuentre todos los elementos integrados en un solo espacio. En este primer momento, el alumno realiza su aprendizaje individualmente interactuando con los materiales y actividades presentadas, posteriormente realiza la conversación interactiva a través del discurso con sus compañeros de clases utilizando las aplicaciones asincrónicas mediadas por la red, asimismo, el grupo es retroalimentado por el docente completando ciclo de la interacción social. El argumento anterior, permite focalizar como se materializa el aprendizaje constructivista en los espacios de formación virtual, de modo que, los esquemas de conocimiento son propiciados en la mente del sujeto y en la actividad de pensamiento. Capacho (2011) afirma que "el enfoque constructivista se fundamenta en el proceso de aprendizaje y no en sus resultados" (pág. 116).

De este modo, el enfoque constructivista aplicado en los ambientes virtuales como el "blearning" y "flipped classroom" propicia el aprendizaje mediante las actividades didácticas mediadas por TIC (Hernández, 2018). Para ello, las tareas o consignas deben ser complejas y representativas acorde a situaciones reales, para que se dé una combinación de la teórica y práctica, así evitar caer en la monotonía de que el espacio virtual solamente sirva como repositorio de contenidos (Morales, 2106; Sánchez, 2017; Sulaimann, 2018).

\section{El aprendizaje invertido en el nivel medio superior}

La estrategia del aprendizaje invertido (AI) ha sido abordada por diversos autores, así como también su uso en los diferentes niveles educativos, desde primaria hasta posgrado, asimismo ha sido implementado para diferentes materias (González, et al., 2017; Merla y Yañez, 2016); basados en este tipo de estudios existen diversas investigaciones de la enseñanza de la segunda lengua (inglés) a nivel bachillerato apoyados mediante el aula inversa, destacando aquellos que promueven el aprendizaje significativo (Akbel, 2018; Basal, 2018; Chivata y Oviedo, 2018); los que se basan en el aprendizaje basado en proyectos (Deng, 2018; Ekmekci, 2018); los que refuerzan la escritura y gramática (Iyitoglu y Erisen, 2018; Sulaiman, 2018).

En el estudio presentado por Quiros (2018) realizó un corte transversal de 85 estudiantes mediando su aprendizaje a través del aula invertida destacando que más del 50\% afirma contraer un compromiso al momento de estudiar con el método del aprendizaje invertido en comparación con el aprendizaje tradicional. Por lo tanto, más del 60\% afirma que mejoraron su aprendizaje del idioma inglés. Martínez-Olvera y Esquivel-Gámez (2018) realizaron un estudio cuantitativo, aplicando el aprendizaje invertido como pedagogía de enseñanza del idioma inglés en educandos de nivel de bachillerato entre los resultados destacan que los alumnos alcanzaron una mejor ejecución en lectura en contraste con el grupo que participo de manera tradicional.

Por su parte la investigación realizada por Calderon (2018) sostiene que el aprendizaje invertido apoyo el desarrollo del vocabulario del idioma inglés en estudiantes de media superior (bachillerato) obteniendo un $90 \%$ que afirma que mejoro su vocabulario utilizando el AI, contra un $60 \%$ que se formó mediante la clase tradicional. Asimismo, los resultados del estudio de 
Calderon (2018) presentaron una diferencia estadísticamente significativa entre los puntajes al momento de realizar la prueba para formar oraciones completas con $85 \%$ de aciertos entre quienes llevaron el aula invertida en comparación del grupo control con un 50\% de aciertos.

En el estudio realizado por Carignano (2016) después de indagar por qué a los estudiantes les costaba trabajo el concentrarse en clases, concluyeron, que era debido a que llegaban cansados después de movilizarse por horas en la ciudad para trasladarse a la escuela. A partir de ello, implementaron la estrategia del Aula Invertida, para que los alumnos aprovecharan el tiempo estudiando los contenidos temáticos en su casa para que a la hora de llegar al salón la dinámica fuera variada. Entre los resultados destacan una diferencia estadísticamente medianamente mayor, entre los promedios de aquellos estudiantes que llevaron la clase mediante apoyados con el aula virtual en contraste con aquellos que tomaron su clase de manera tradicional; los resultados fueron catalogados de la siguiente manera: a) Conocimiento $+2.98 \%$, b) desempeño $+6.90 \%$, c) producto $+10.65 \%$ y d) evaluación $+4.66 \%$.

Por su parte el estudio realizado por Ekmekci (2018) investigó el impacto del aula invertida en el aprendizaje de la segunda lengua (inglés) utilizando la herramienta Edmodo como Aula Invertida, de los resultados destacan que los estudiantes apoyados en el AI obtuvieron una media en la desviación estándar de 71,49 en comparación con el grupo control $(58,30)$ con respecto a las habilidades adquiridas para la escritura del idioma inglés. Asimismo, las percepciones fueron positivas (80\%) en la aceptación de la herramienta Edmodo como ambiente virtual de aprendizaje.

De acuerdo con el artículo de Orhan (2018) realizó un estudio en estudiantes de bachillerato para el aprendizaje del idioma inglés utilizando el modelo de aula invertida o aprendizaje inverso, en dicho estudio se evaluaron cuatro habilidades (lectura, escritura, gramática y escucha), los resultados encontrados apuntan que los alumnos del grupo experimental (AI) presentaron diferencias estadísticas significativas con respecto al grupo control (clase tradicional). Las conclusiones del estudio enfatizan que el aprendizaje mediante el AI permite el acceso al educando a herramientas tecnológicas en interacción con sus pares y que además el material suministrado en la plataforma virtual promueve el auto aprendizaje del alumnado.

Tal como se aprecia en párrafos anteriores, desde esta metodología los discentes se responsabilizan de sus aprendizajes, configurándose en entes responsables de sus contextos educativos, el mediar el aprendizaje con las TIC no es algo nuevo, aquí resalta la didáctica empleada al "invertir" el modelo educativo tradicional mediando el aprendizaje desde otro enfoque (May y Tutyub, 2017). Desde esta postura axiológica se vislumbra que el aprendizaje invertido promueve el diseño de programas académicos y mediarlos en entornos semi presenciales. En este sentido, el aprendizaje invertido propicia la construcción de los procesos cognitivos de cada estudiante asignándole la responsabilidad de su quehacer educativo (Herrera y Prendes, 2019; Hinojo et al., 2019).

Por lo tanto, la mediación pedagógica del aprendizaje invertido proporciona experiencias educativas enriquecedoras, trasladando las actividades individuales a espacios virtuales para aprovechar al máximo la clase presencial detonando la interacción cara a cara entre docentes y discentes con el fin de propiciar las posibilidades en la construcción del conocimiento (Hu, 2019; Iyitoglu y Erisen, 2018; Leis y Brown, 2018). 
Lo anterior, es derivado de trasladar las teorías y actividades en el entorno virtual para ser consultados previos a la sesión presencial lo que posibilita la realización de prácticas de aprendizaje tales como: exposiciones, debates, ejercicios, proyectos, fomentando la colaboración, e interacción entre estudiantes. Con base en lo anterior, el objetivo fue analizar la contribución del aula invertida como estrategia de enseñanza del idioma inglés, teniendo como pregunta de investigación ¿qué diferencia existe entre las metodologías del aprendizaje inverso y el aprendizaje tradicional en relación al desempeño académico de los estudiantes de bachillerato?

\section{METODOLOGÍA}

El presente estudio es de corte cuantitativo descriptivo con diseño cuasi-experimental, cuya variable del estudio se basa en el aprovechamiento académico en la asignatura de lengua adicional al español (inglés I) de un grupo de estudiantes que cursaron el primer semestre de un bachillerato público en la modalidad mixta. Para lo anterior se utilizó dos grupos, un grupo experimental y otro de control. Cabe precisar que los sujetos fueron elegidos de manera no aleatoria accidental, derivado de que los grupos ya estaban conformados antes del inicio del experimento. Para la valoración del impacto de la intervención experimental, se analizaron las calificaciones de ambos grupos como evidencia de su desempeño académico.

\section{Población}

El criterio de la muestra se definió como no probabilístico y muestreo por conveniencia derivado de la accesibilidad a la población objeto de estudio, siendo factores relevantes para el estudio (Hernández, Fernández y Baptista, 2014). El escenario en el cual se desarrolló esta investigación comprende la asignatura de lenguaje adicional al español (inglés I) correspondiente al bachillerato inscritos en el semestre lectivo 2018-2019 en la modalidad mixta, en el que los estudiantes solo acuden un día a la semana (sábado) para tomar sus clases de manera presencial.

\section{Instrumentos}

Para estimar el efecto en la intervención educativa del aprendizaje invertido mediante el desempeño académico, una vez terminado el curso, se procedió a valorar los resultados de las calificaciones logrados por cada uno de los participantes de ambos grupos. Asimismo, se aplicó una encuesta piloto al grupo experimental para obtener sus apreciaciones con respecto al aprendizaje invertido. Finalmente, se realizó una entrevista a la docente que impartió las clases a ambos grupos, para conocer su perspectiva en torno a la enseñanza mediante el aprendizaje invertido.

\section{Aprendizaje invertido del inglés}

La investigación se desarrolló en la asignatura de lenguaje adicional al español (inglés I), el objetivo final de la asignatura es proporcionar los conocimientos para desarrollar la capacidad de comunicarse en un nivel de dominio intermedio de acuerdo a estándares internacionales del Marco Común Europeo de Referencia a las Lenguas. Lo anterior, se propició mediante el desarrollo de las habilidades comunicativas: comprensión auditiva, comprensión lectora, expresión e interacción oral y expresión escrita. Para ello, se aplicó la metodología de Aula Invertida (AI) en donde el contenido temático se trasladó a una plataforma digital educativa actualizándose semanalmente. 
En dicha plataforma se pusieron los recursos educativos para el trabajo autónomo de cada clase tales como videos, infografías, actividades de aprendizaje y autoevaluaciones correspondientes para cada clase.

En cuanto a la interacción la comunicación se realizó por medio del Foro, en el cual los estudiantes realizaron sus aportaciones en el idioma inglés; asimismo, la retroalimentación por parte del docente se realizó en el mismo idioma. La distribución del contenido temático se desarrolló con base el programa de estudio de la asignatura, en la Tabla 1, se muestran los objetivos de aprendizaje realizados durante el curso.

Posteriormente, en el aula, se realizaron distintas estrategias que propiciaron el trabajo colaborativo entre estudiantes para la aclaración de dudas. Para ello, se aplicó lo aprendido a través del desarrollo de consignas que servían como evidencia palpable del desarrollo de los estudiantes.

Tabla 1. Objetivos desarrollados por semana, elaboración propia.

\begin{tabular}{|c|c|}
\hline Semana & Objetivos \\
\hline Primera & $\begin{array}{l}\text { - Conocer una forma de presentarse y presentar a los demás. } \\
\text { - Familiarizarse con vocabulario relacionado a miembros de la familia. } \\
\text { - Concientizar el uso gramatical del verbo ser o estar en inglés. }\end{array}$ \\
\hline Segunda & $\begin{array}{l}\text { - } \quad \text { Complementar vocabulario para expresar información personal. } \\
\text { - Analizar situaciones en las que se comparte información personal. } \\
\text { - } \quad \text { Expresar información personal escrita y oralmente. }\end{array}$ \\
\hline Tercera & $\begin{array}{l}\text { - Identificar actividades que se realizan de manera rutinaria. } \\
\text { - Reflexionar sobre diferentes rutinas diarias. } \\
\text { - Reconocer la estructura gramatical para expresar rutinas diarias. }\end{array}$ \\
\hline Cuarta & $\begin{array}{l}\text { - Identificar la frecuencia en la que se realizan actividades comunes. } \\
\text { - Practicar la estructura gramatical del presente simple para expresar rutinas } \\
\text { saludables. } \\
\text { - Concientizar sobre distintos estilos de vida. }\end{array}$ \\
\hline Quinta & $\begin{array}{l}\text { - Reconocer actividades que se realizan al tiempo en que se habla. } \\
\text { - Identificar estructura gramatical del presente continuo. }\end{array}$ \\
\hline Sexta & $\begin{array}{l}\text { - Identificar sustantivos, relativos a comida, contables e incontables. } \\
\text { - } \quad \text { Concientizar sobre el respeto a la cultura de la comida de diferentes lugares. }\end{array}$ \\
\hline
\end{tabular}

La plataforma utilizada para el aprendizaje invertido fue Moodle (ver figura 1), en donde se alojaron las didácticas de aprendizaje que los estudiantes tenían que desarrollar. Cada tema estaba caracterizado por los objetivos a realizar mediante un contenido principal denominado clase, que explicaba los objetivos a alcanzar, los contenidos incluían los aspectos teóricos conceptuales, vídeos explicativos, material de lectura y hojas de trabajo cuya finalidad era practicar y desarrollar la lectura y escritura del idioma inglés a través de ejercicios complementarios. 


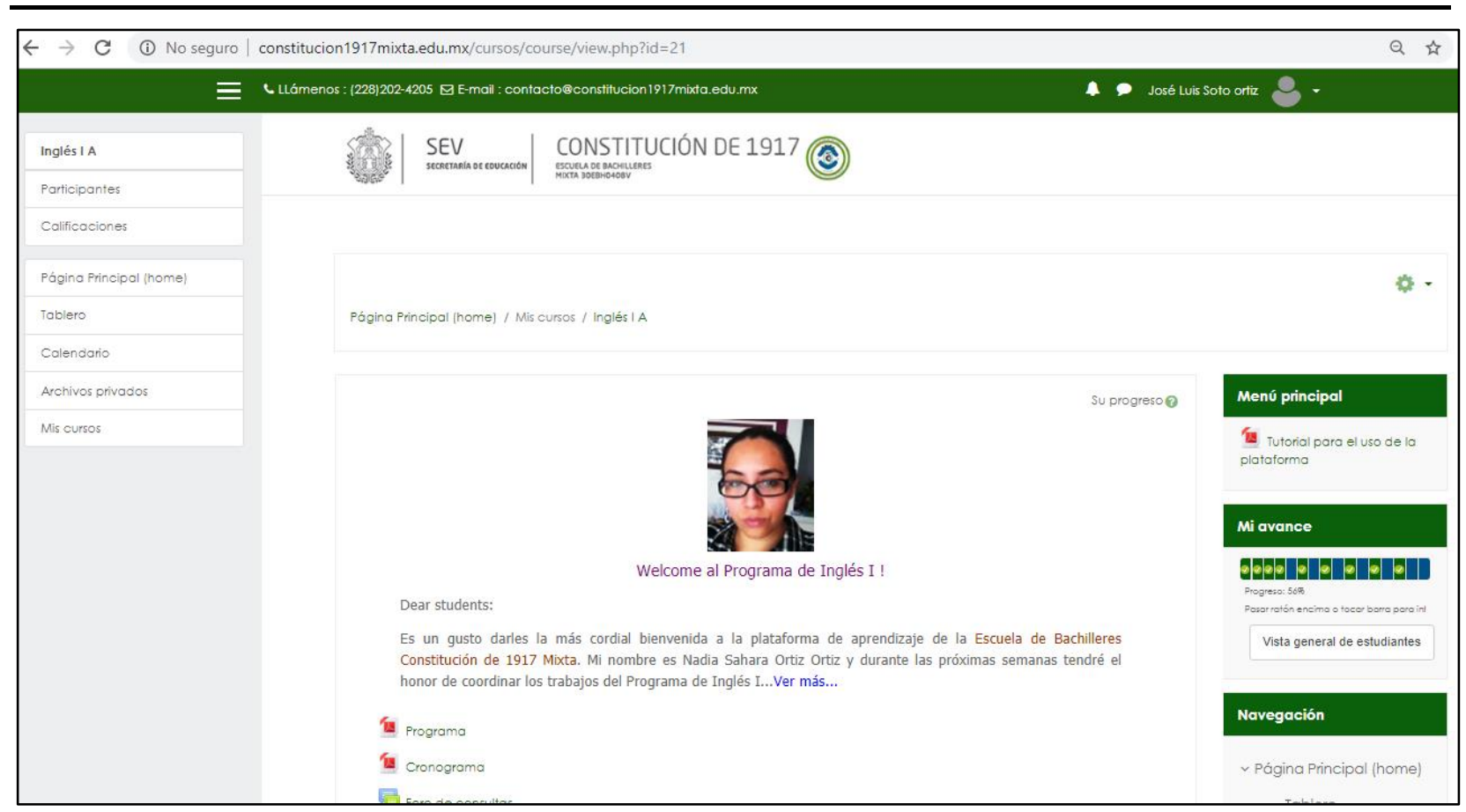

Figura 1. Plataforma tecnológica del aprendizaje invertido, elaboración propia.

\section{Procedimiento}

Grupo experimental (G1). Previo al inicio del curso de lengua adicional al español (inglés I), se diseñó un sitio web denominado "Constitución de 1917" al que podían tener acceso los alumnos de la modalidad de aprendizaje invertido. El diseño instruccional se ajustó para adaptarlo tomando los objetivos y contenidos temáticos utilizados en la clase tradicional tal como se estipula en el plan de estudios. De manera paralela, y antes de iniciar el curso lectivo, se capacitó a los estudiantes en el manejo de la plataforma tecnológica, dicha instrucción permitió que los discentes aprendieran el ingreso, actualizar sus datos, conocer la ubicación del tablón del curso, de los foros y del acceso al material de consulta, distribuido por clases semanales.

Para el contenido conceptual, la asignatura se organizó en semanas, dosificando la temática mediante "clases" que incluían el análisis de archivos multimedia, la visualización de presentación de diapositivas, una autoevaluación de la semana, y una hoja de trabajo para la elaboración de ejercicios en formato Word, a los que los estudiantes debían consultar previamente y llevar impreso para su discusión en las sesiones presenciales, las cuales se llevaron a cabo una vez por semana los días sábados.

Grupo Control (G2). El curso se impartió de manera tradicional las seis semanas que duro el curso, la didáctica instruccional para la impartición de las clases fue la que normativamente se emplea de acuerdo con el plan y programa de estudios, misma que consistió en sesiones explicativas por parte del docente con pequeños ejercicios en las sesiones presenciales las cuales se llevaron a cabo una vez por semana los días sábados. Al igual que en el grupo experimental, el grupo control, realizó los ejercicios de la hoja de trabajo en formato Word, con la diferencia de que estos fueron suministrados por el docente en cada clase a los estudiantes. 
En ambos Grupos se trabajaron los mismos contenidos y los ejercicios programados para las sesiones presenciales, al final del curso se evaluaron las actividades, se aplicó el examen final y se realizaron las encuestas. Los resultados fueron procesados en el SSPS versión 18.

\section{RESULTADOS}

Tomando como referente la pregunta de investigación ¿qué diferencia existe entre las metodologías del aprendizaje inverso y el aprendizaje tradicional en relación al desempeño académico de los estudiantes de bachillerato? en la Tabla 2 se muestra la comparación de los resultados de las medias de calificaciones (Consignas y Final) de ambos grupos utilizando la prueba estadística t de student, en la que se observa un nivel de significancia de 0,024, lo cual, al ser menor de .05 indica que existen diferencias significativas entre el grupo experimental y el control.

Tabla 2. Resultados de la muestra de la prueba t de student, elaboración propia.

\begin{tabular}{llccc}
\hline & & statistic & df & p \\
\hline Final & Student's t & 2.43 & 22.0 & 0.024 \\
Consignas & Student's t & 3.55 & 22.0 & 0.002 \\
\hline
\end{tabular}

De acuerdo con lo anterior, se describe los resultados de ambos grupos, con respecto al grupo control quien recibió la instrucción educativa de manera tradicional presentó una media de 5.25 en la calificación final comparado con el grupo experimental que recibió la intervención del aprendizaje invertido quien tuvo una media de 7,17 en la calificación final, por lo que se observa que tuvieron un promedio mayor el G1 con respecto a G2. Lo mismo sucede con respecto a las consignas realizadas por parte de los estudiantes, los resultados obtenidos en una escala del 0 al 6 , ya que fueron las tareas extra-clase que debían realizar la media obtenida por el G2 (control) fue menor 3.00 con respecto a la media obtenida por el G1 (experimental) que fue de 4.33 (ver Tabla $3)$.

Tabla 3. Estadísticos descriptivos organizados por grupos, elaboración propia.

\begin{tabular}{llccccc}
\hline & Group & $\mathbf{N}$ & Mean & Median & SD & SE \\
\hline \multirow{2}{*}{ Final } & Grupo1 & 12 & 7.17 & 7.00 & 0.937 & 0.271 \\
& Grupo2 & 12 & 5.25 & 6.00 & 2.563 & 0.740 \\
Consignas & Grupo1 & 12 & 4.33 & 4.00 & 0.985 & 0.284 \\
& Grupo2 & 12 & 3.00 & 3.00 & 0.853 & 0.246 \\
\hline
\end{tabular}


En relación con los resultados obtenidos en la encuesta realizada en el G1 para obtener sus apreciaciones con respecto a la intervención educativa bajo la modalidad del aprendizaje invertido, los resultados fueron organizados en tres variables: a) dinámica del curso, b) actividades realizadas, y c) retroalimentación por parte del docente. En este sentido, en la primera variable, más del $60 \%$ señala que la dinámica del curso, así como el contenido temático en cuanto al acceso y diseño fueron calificados como "muy buenos.

Con respecto a la segunda variable más del $71 \%$ afirma que las actividades realizadas les permitieron practicar, comprender y aprender el idioma inglés, pues en dichas didácticas fueron fáciles de elaborar. Finalmente, de acuerdo con la variable relacionada con la retroalimentación, los resultados destacan que más de $68 \%$ confirmó que el docente respondió en tiempo y forma las dudas plasmadas en los foros de consulta, así mismo el seguimiento realizado por el docente los motivó y oriento en la resolución de los cuestionamientos realizados mediante mensajes directos.

Con base en la entrevista semiestructurada dirigida al docente que impartió el curso, al cuestionarle acerca de sus apreciaciones con respecto a la enseñanza mediante la modalidad del aprendizaje invertido, al cuestionarle acerca de su opinión con respecto a la metodología que se utilizó para la realización de curso de inglés, señaló lo siguiente: “Tengo una opinión positiva pues la metodología de aula inversa me resultó renovadora y refrescante para mi práctica docente, especialmente, en el contexto del bachillerato mixto. En este, contamos con pocas estrategias que combatan la carencia de tiempo, espacio y recursos tecnológicos. Encontré en esta metodología la forma de ahorrar tiempo al capacitar al alumno con anticipación, desglosando la teoría de la materia de forma accesible y considerando las oportunidades de cada uno de ellos".

Al plantear el cuestionamiento ¿Cuál es tu percepción con respecto a la experiencia desde tu práctica docente? Su respuesta fue la siguiente: "Implementar la metodología de aula inversa, ha sido uno de los mejores recursos que he podido experimentar en la preparatoria mixta. Teniendo en cuenta que en este contexto educativo carecemos, como ya mencioné previamente, de tiempo, de espacios y también de tecnología pude comprobar que al aplicar la metodología de aula inversa obtuvimos (maestro y alumnos) varios beneficios. Estos incluyen: Los estudiantes pueden realizar sus clases en una plataforma educativa a su propio ritmo, en su propio espacio, al momento que ellos mismos programen su horario de trabajo. Como maestra pude utilizar otros recursos que normalmente me quitan tiempo en clase pero que a los alumnos les llama más la atención como videos, presentaciones ppt o infografías.

Los alumnos también tuvieron la oportunidad de medir sus propios avances al realizar una autoevaluación semanal, lo cual a mi me ayudó a tener un mayor control de quien está avanzando y quien se ha quedado atrás. Gracias a que los alumnos son capacitados con la teoría en la plataforma, obtuve suficiente tiempo para organizar actividades significativas que promueven el trabajo colaborativo en el aula. Pude monitorear las actividades que realizaban de manera más práctica y organizada. Los alumnos parecían más relajados durante clase y motivados a trabajar pues ya tenían conocimiento del tema y dominio de las actividades.

También considero algunos aspectos negativos que tenemos que afrontar como por ejemplo los alumnos que no tienen disponible un equipo, un espacio o una red para conectarse y trabajar. Por esto propongo que las clases se publicaran el mismo sábado para que los alumnos que tienen complicaciones para trabajar entre semana puedan hacerlo desde el sábado y tengan 
disponible la sala de cómputo de la escuela. También considero que los alumnos necesitan ser motivados constantemente para no parar de trabajar en la plataforma, ya que ellos deben hacerse responsables de su propio aprendizaje y muchos no están acostumbrados a la forma de trabajo. Tener comunicación constante con los alumnos es también complicado pues entre semana ambos tenemos actividades diferentes. Pude observar que al perder comunicación con los alumnos ellos también pierden interés por el trabajo en plataforma".

Asimismo, al preguntar al docente sí considera que el desempeño académico de tus estudiantes mejoró con el método de aprendizaje invertido con respecto a la clase tradicional, explica a detalle el ¿por qué? "Considero que hubo una diferencia importante en los avances de los alumnos, como resultado de la implementación de la metodología. Los resultados que pude observar fueron: Me parece que los estudiantes encontraron las clases de la plataforma más interesantes y accesibles para su aprendizaje. A muchos alumnos les agradaba obtener un puntaje alto en sus autoevaluaciones y tuvieron las oportunidades para lograrlo, esto también les ayudó para subir su calificación final. Al tener los datos de la plataforma a la mano, pude presionar a los alumnos que no ingresaban o se atrasaban cada semana y así comprometerlos a esforzarse más para mejorar su rendimiento escolar. Los alumnos tenían mayor confianza a la hora de realizar la práctica dentro del aula.

La mayoría realizaba las actividades previas en plataforma y presentaban resultados en el aula. La práctica tiene un valor mayor en la calificación integral de los alumnos, por lo que, si no faltaban a clases, realizaban evidencias sustanciales de su aprendizaje y de forma colaborativa con las que obtuvieron mayor puntaje al final del programa. Los alumnos tuvieron tiempo suficiente para organizarse y trabajar sin ninguna prisa ni presión y siempre con guía de su maestra. Por estas razones considero que con la implementación de la metodología se obtuvieron mejores resultados finales de los alumnos".

Finalmente, al docente se le cuestionó acerca de sus percepciones con respecto a la utilización de la plataforma en este sentido ¿Cuál fue la mayor dificultad que tuvo para impartir este curso? la mayor dificultad es comenzar. Cambiar en $180^{\circ}$ la dinámica del programa. Poder integrar recursos llamativos para los alumnos y también apropiados. Diseñar las clases en la plataforma de manera fluida y en armonía con las clases en el aula, sin olvidar los objetivos del programa y las necesidades de los alumnos. Asimismo, al cuestionar acerca de la facilidad de acceso ¿Qué facilidad tiene para acceder a la plataforma virtual desde casa o en algún otro lugar? "toda la facilidad en cuanto a lo técnico pues solo se necesita un drive e internet. Sin embargo, coordinarse en horarios fuera del horario de trabajo es a veces dificil".

\section{DISCUSIÓN Y CONCLUSIONES}

Los resultados presentados, reflejan un mayor aprovechamiento en el desempeño académico por parte de los estudiantes al someterse en la modalidad del aprendizaje invertido en comparación con los discentes que llevaron la clase tradicional. En este sentido, se confirma lo planteado por Ekmekci (2018) quien afirma que la mediación del proceso del aprendizaje con el modelo de aprendizaje invertido propicia el reforzamiento en el desempeño académico en los estudiantes. En este sentido, el grupo experimental mostro mejores promedios en comparación con el grupo 
control. Asimismo, en la entrega de tareas los estudiantes del grupo experimental entregaron sus actividades contrario al grupo control, en algunos estudiantes no entregaron el total de las consignas solicitadas como parte de su formación en el idioma inglés.

Entre las ventajas encontradas destacan, que los estudiantes se mostraron motivados con el material presentado en la plataforma virtual, ya que les permitió el reforzamiento de la teoría con el desarrollo de la multimedia presentada, otro de los aspectos importantes fue la retroalimentación por parte del docente quien estuvo disponible entre semana para la aclaración de dudas por parte de los estudiantes, es de resaltar lo anterior puesto que la contratación del docente solamente se enfoca en asistir en el horario destinado para su sesión presencial.

Una de las limitantes, en específico con el modelo educativo del bachillerato objeto de estudio es la propia modalidad mixta, en donde los estudiantes por normativa solamente acuden un día a la semana para asistir a sus sesiones magistrales. Otro aspecto, es la diversidad en la condición personal de los estudiantes, ya que la mayoría realiza sus estudios bajo esta modalidad por motivos laborales o bien por su situación económica solamente así pueden cursar su bachillerato. En cuanto al aspecto didáctico un limitante que se encontró, fue en relación con la dinámica de los foros, pues estos fueron diseñados para la atención de dudas, por lo que se debe de considerar una mayor interacción entre los estudiantes y no solamente focalizado en el docente, promoviendo así una interacción colaborativa.

A manera de cierre, es cierto que las experiencias educativas en el extranjero, así como a nivel nacional con respecto al aprendizaje invertido han ido creciendo de manera paulatina. Sin embargo, a nivel regional falta mucho trabajo por realizar, en el caso de la modalidad del bachillerato mixto, ofrece una ventana de oportunidad para la implementación de modelos educativos mediados por TIC, y que sin lugar a dudas los modelos virtuales de aprendizaje se adaptan perfectamente dadas las características propias de la escuela. Asimismo, se resalta, que falta un cambio en la cultura del docente, debido ante la renuencia por utilizar este tipo de didácticas de enseñanza, deduciendo que es por el tiempo que deben invertir fuera de su horario laboral. Finalmente, la presente investigación pone de manifiesto la posibilidad de aplicar el aprendizaje invertido en los bachilleratos públicos en el estado de Veracruz, lo que propiciaría el reforzamiento de los aprendizajes adquiridos en el aula por parte de los estudiantes.

\section{REFERENCIAS BIBLIOGRÁFICAS}

Aguirre, L. (2017). El uso del aula invertida en el autoaprendizaje del estudiante. Propuesta: Diseño de una guía instruccional (Tesis de posgrado). Universidad de Guayaquil, Colombia. Recuperado de: http://repositorio.ug.edu.ec/handle/redug/25413

Akbel, B. (2018). Student's and instructor's opinions on the implementation of Flipped Learning Model for cello education in Turkish music. Journal of Education and Trianing Studies, Vol. 6, No. 8, doi: 10.11114/jets.v6i8.3256, Recuperado de: https://eric.ed.gov/?id=EJ1181956

Asens, M. (2015). El modelo de aprendizaje invertido como herramienta innovadora en la asignatura de empresa e iniciativa emprendedora de formación profesional (tesis de 
grado). Universidad Internacional de la Rioja, España. Recuperado de: https://reunir.unir.net/handle/123456789/2971

Basal, A. (2018). The implementation of a Flipped Classroom in foreign language teaching. Turkish Online Journal of Distance Education, Vol. 16 No. 4. Recuperado de: https://eric.ed.gov/?id=EJ1092800

Bergmann, J., Sams, A. \& cols. (2014) What Is Flipped Learning? Flipped Learning Network (FLN). Recuperado de:

http://www.flippedlearning.org/cms/lib07/VA01923112/Centricity/Domain/46/FLIP_han dout_FNL_Web.pdf

Bloom, B. (1973). Taxonomía de los objetivos de la educación. Buenos Aires: El Ateneo.

Capacho, J. (2011). Evaluación del aprendizaje en espacios Virtuales-TIC. Editorial Universidad del Norte. España.

Caraguay, E. (2017). Estrategia educativa para potenciar el aprendizaje de los alumnos de primer año de bachillerato del colegio "San Francisco" de la provincia de Zamora Chinchipe en el año 2016 (tesis de magister). Universidad de Loja, Ecuador. Recuperado de: http://dspace.utpl.edu.ec/handle/20.500.11962/20803

Cerda, N. (2014). los recursos audio-visuales para el aprendizaje del idioma inglés en los estudiantes de primer año de bachillerato de la unidad educativa fisco misional Fray Bartolomé De Las Casas de la comunidad indígena "Los Salasacas" de la provincia de Tungurahua (tesis de grado). Universidad Técnica de Ambato. Ambato, Ecuador. Recuperado de: http://repositorio.uta.edu.ec/jspui/handle/123456789/8894

Chivata, Y. y Oviedo, R. (2018). EFL student's perceptions of activeness during the implemention of flipped learning approach at a Colombian University. Gist Education and Learning Research Journal, No. 17. Recuperado de:

https://dialnet.unirioja.es/servlet/articulo?codigo $=6758706$

Deng, L. (2018). The Project-based flipped learning model in business english translation course: Learning, teaching and assesment. Englihs Language Teaching, Vol. 11, No. 9.

Recuperado de: https://eric.ed.gov/?id=EJ1188576

Ekmekci, E. (2018). The flipped writing classroom in Turkish EFL context: A comparative study on a niew model. Turkish Online Journal of Distance Education, Vol. 18. No. 2.

Recuperado de: https://eric.ed.gov/?id=EJ1145315

González, C., Fleito, I., González, M., Valdunciel, L., Álvarez, J. y Sarmiento, A. (2017). Modelo de enseñanza-aprendizaje basado en la clase invertida: una experiencia de innovación docente en diferentes niveles educativos. Revista Infancia Educación y Aprendizaje, Vol. 3. No. 2. Recuperado de: https://revistas.uv.cl/index.php/IEYA/article/download/700/675

González, M. y Huerta, P. (2019). Experiencia del aula invertida para promover estudiantes prosumidores del nivel superior. RIED. Revista Iberoamericana de Educación a Distancia, 22(2), pp. 245-263. doi:10.5944/ried.22.2.23065

Hernández, A. (2018). El modelo IMSCI como estrategia de acompañamiento para mejorar la redacción en inglés con alumnos del bachillerato Enrique Benítez (tesis de maestría). Benemérita Universidad Autónoma de Puebla, Puebla. Recuperado de: https://repositorioinstitucional.buap.mx/handle/20.500.12371/1002 
Hernández, R., Fernández, C. y Baptista, P. (2014). Metodología de la investigación (6 ${ }^{\mathrm{a}}$ ed.). México: McGrwall Hill Education.

Herrera, G. y Prendes, M. (2019). Implementación y análisis del método de aula invertida: un estudio de caso en Bachillerato. Revosta INNOEDUCA. Vol. 5, No. 1. doi:1024310/innoeduca.2019v5i1.3091

Hinojo Lucena, f. J.; Aznar díaz, I.; Romero Rodríguez, J. M.; Marín Marín, J. A. (2019). Influencia del aula invertida en el rendimiento académico. Una revisión sistemática. Campus $\quad$ Virtuales, $8(1), \quad 9-18 . \quad$ Recuperado de: http://uajournals.com/ojs/index.php/campusvirtuales/article/view/384

$\mathrm{Hu}$, Ch. (2018). The value of adding a flipped learning component to a humanities course in higher education: student perception and perfomance. Taiwan Journal of TESOL, Vol. 15.2, doi:10.30397/TJTESOL.201810_15(2).0001. Recuperado de: https://eric.ed.gov/?id=EJ1195408

Iyitoglu, O. y Erisen, Y. (2018). Delving into Flipping EFL Classroom: A mixed method study. European Journal of English Language Teaching, Vol. 3. Issue 1. doi:10.5281/zenodo.1045310. Recuperado de: https://eric.ed.gov/?id=ED590461

Leis, A. y Brown, K. (2018). Flipped learning in an EFL environment: Does the teacher's experience affect learning outcomes? The EUROCALL Review, Vol. 26, No. 1. Recuperado de: https://polipapers.upv.es/index.php/eurocall/article/view/8597/10476

Martínez-Olvera, W. y Esquivel-Gámez, I. (2018). Uso del modelo del aprendizaje invertido en un bachillerato público. RED. Revista de Educación a Distancia. Núm. 58, Artíc. 11, 31 10-2018. doi:10.6018/red/58/11.

May, N. y Tuyub, T. (2017). Estrategia para disminuir el índice de reprobación en la unidad de aprendizaje de inglés en una institución de nivel medio superior. Revista Electrónica del Desarrollo Humano para la Innovación Social. Vol. 4. Núm. 8. Recuperado de: http://www.cdhis.org.mx/index.php/CAGI/article/view/123

Merla, A. y Yañez. C. (2016). El aula invertida como estrategia para la mejora del rendimiento académico. Revista Mexicana de Bachillerato a Distancia. Núm. 16. Año 8. Recuperado de: http://www.revistas.unam.mx/index.php/rmbd/article/view/57108

Morales, A. (2016). Técnicas de estudio en el aprendizaje gramatical del idioma inglés en los estudiantes de décimo educación general básica paralelos “A, B, C, D” del Colegio Nacional "Hipatia Cárdenas de Bustamante" período 2015- 2016, (tesis de magister). Universidad Central del Ecuador, Ecuador. Recuperado de: http://www.dspace.uce.edu.ec/handle/25000/12375

Quintanilla, J. (2016). Las plataformas virtuales libres y el aprendizaje significativo del idioma inglés en los estudiantes de la unidad educativa Hispano América (tesis de magister). Universidad Técnica de Ambato. Ambato, Ecuador. Recuperado de: http://repositorio.uta.edu.ec/jspui/handle/123456789/21377

Sánchez, R. (2017). Aula invertida, metodología del siglo XXI (tesis de magister). Universidad de las Islas Baleares, España. Recuperado de: http://dspace.uib.es/xmlui/handle/11201/147021 
Sulaiman, N. (2018). Implementing blended learning and flipped learning models in the university classroom: a case study. Teaching English With Technology. Vol 18, No. 4. Recuperado de: https://eric.ed.gov/?id=EJ1195820 